\title{
COVID - 19 Presenting with Fungal Infections - The Hidden Pandemic within Pandemic?
}

\author{
Vidyashree S. Hulkoti ${ }^{1}$, Samarth Shukla ${ }^{2}$, Sourya Acharya ${ }^{3}$, Dhruv Talwar ${ }^{4}$, Aditi Goyal ${ }^{5}$ \\ 1, 2, 3, 4, 5 Department of Medicine, Datta Meghe Institute of Medical Sciences, \\ Sawangi (Meghe), Wardha, Maharashtra, India.
}

\section{INTRODUCTION}

As the pandemic continues to spread vigorously, it is being noted that the COVID 19 virus is associated with various complications during the disease and also a great deal of post disease sequel. ${ }^{1}$ These gruelling complications are integrated with the overwhelming infection caused by the cytokine storm produced by the virus. While the treatment modalities are still under trial, glucocorticoids seem to have played a pivotal role in putting a check to the inflammation caused by the virus and have forbidden the organ damage caused thereafter.

However, the aftermath of glucocorticoids usage has its own benefits and risks. Glucocorticoids cause cellular immunodeficiency and thus have immunosuppressive effects, additionally the use of immunomodulators such as tocilizumab alters the immune system and it subsequently predisposes the host to various secondary opportunistic infective agents. In the current state, as the pandemic abstains from fading away, an increasing trend of secondary fungal infections has been seen with COVID-19, resulting in an outbreak of fungal infections such as mucormycosis and candidiasis. Mucormycosis refers to any infection caused by the fungi of the Order Mucorales. Mucormycosis has been documented in the literature to be associated with a high rate of mortality due to its potential to spread drastically. ${ }^{2}$

Altered immunity is an important risk factor for mucormycosis. Additionally, diabetes has been noted to be critical for the development of mucormycosis in immunocompetent patients. Candidiasis is an infection caused by the candida species due to the immunosuppressed state developed by the use of glucocorticoids, which results in secondary fungal infection requiring urgent medical attention. The objective of this case report is to highlight the impending secondary fungal infection outbreak in COVID-19 and the need to contain this emerging spread of fungal infections under the blanket of this deadly pandemic.

\section{PRESENTATION OF CASE}

\section{Case 1}

A 48 - year - old female, came with chief complaints of fever for 5 days associated with cough and breathlessness for 2 days. The patient had a history of newly diagnosed diabetes mellitus in the last 1 year and was on oral antidiabetic drugs for the same. There was no history of prior hypertension, malignancy or ingestion of any immunosuppressants. On admission, Pulse - 96 / min, BP - 100 / $70 \mathrm{mmHg}$ in right arm supine position, Spo2 - $88 \%$ on room air, immediate rapid antigen testing for COVID-19 was done which turned out to be positive.
Corresponding Author: Dr. Samarth Shukla, Department of Pathology, Datta Meghe Institute of Medical Sciences, Sawangi (Meghe), Wardha, Maharashtra, India. E-mail: samarth21174@gmail.com

DOI: $10.14260 / \mathrm{jemds} / 2021 / 770$

How to Cite This Article:

Hulkoti VS, Shukla S, Acharya S, et al. COVID-19 presenting with fungal infections - the hidden pandemic within pandemic? J Evolution Med Dent Sci 2021;10(44):38103814, DOI: 10.14260/jemds/2021/770

Submission 09-06-2021,

Peer Review 03-08-2021,

Acceptance 10-08-2021,

Published 30-11-2021.

Copyright (c) 2021 Vidyashree S. Hulkoti et al. This is an open access article distributed under Creative Commons Attribution License [Attribution 4.0 International (CC BY 4.0)] 
High-resolution computed tomography (HRCT) thorax done suggestive of CT score of 16 / 25 and CORADS - 6, nasopharyngeal swab RT - PCR done was positive.

Laboratory investigations were done and the blood panel was suggestive of raised inflammatory markers. The patient was immediately started with supplemental oxygen by face mask, injection remdesivir, antibiotics and other supportive management. Because of the severe category HRCT score, the patient was started on low dose glucocorticoids (Inj. dexamethasone $6 \mathrm{mg}$ once a day) and tapered promptly. As sugar levels were uncontrolled, the patient was started on rapid acting insulin and dose was adjusted according to sugar levels. Gradually the patient's condition improved.

After 5 days of hospital stay, the patient started to complain of pain in the right eye associated with headache and watering of the eye and also a small lesion on the right cheek which gradually turned necrotic. On examination, mild proptosis was noticed on the right side. The patient also complained of a painful lesion on the hard palate which on examination was a whitish plaque. With the suspicion of fungal infection, the patient was started on antifungal injection liposomal amphotericin B $5 \mathrm{mg} / \mathrm{kg}$ / day immediately.

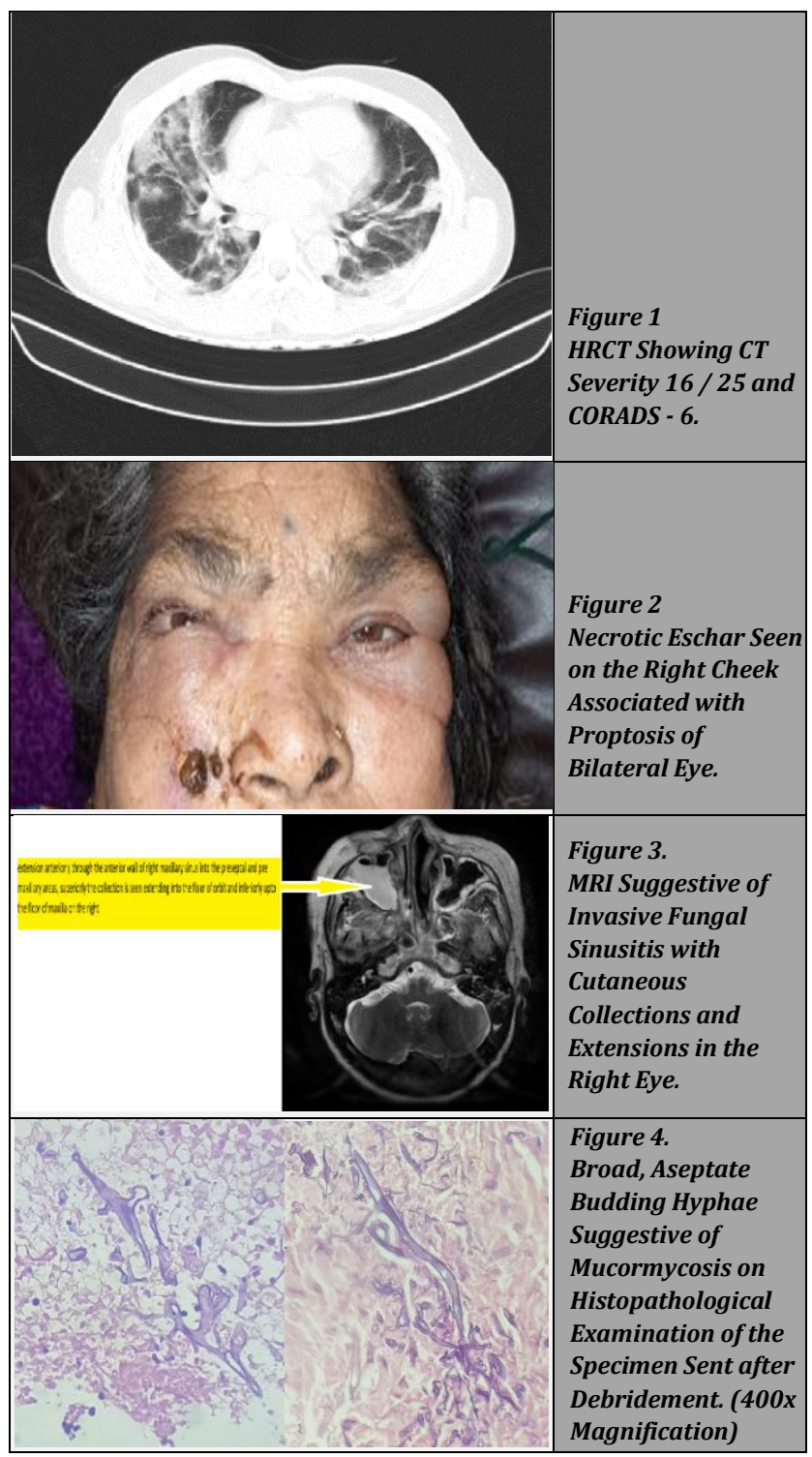

MRI paranasal sinus with orbit was planned, MRI was suggestive of bilateral (right more than left) invasive fungal sinusitis with cutaneous collections and extension anteriorly through the anterior wall of right maxillary sinus into the pre - septal and premaxillary areas, superiorly the collection was seen extending into the floor of the orbit and inferiorly up to the floor of the maxilla on the right.

Debridement of the cheek lesion was done and sent for histopathology, where sections were showed distinct broad, aseptate hyphae, budding at places clearly, suggestive of Mucormycotic pathology.

The patient was diagnosed as having secondary fungal infections and was given anti-fungal liposomal amphotericin $B$ for 21 days along with regular KFT monitoring and hydration.

The patient improved after 21 days and was discharged on oral antifungal posaconazole $200 \mathrm{mg}$ QID and other supportive management.

\section{Case 2}

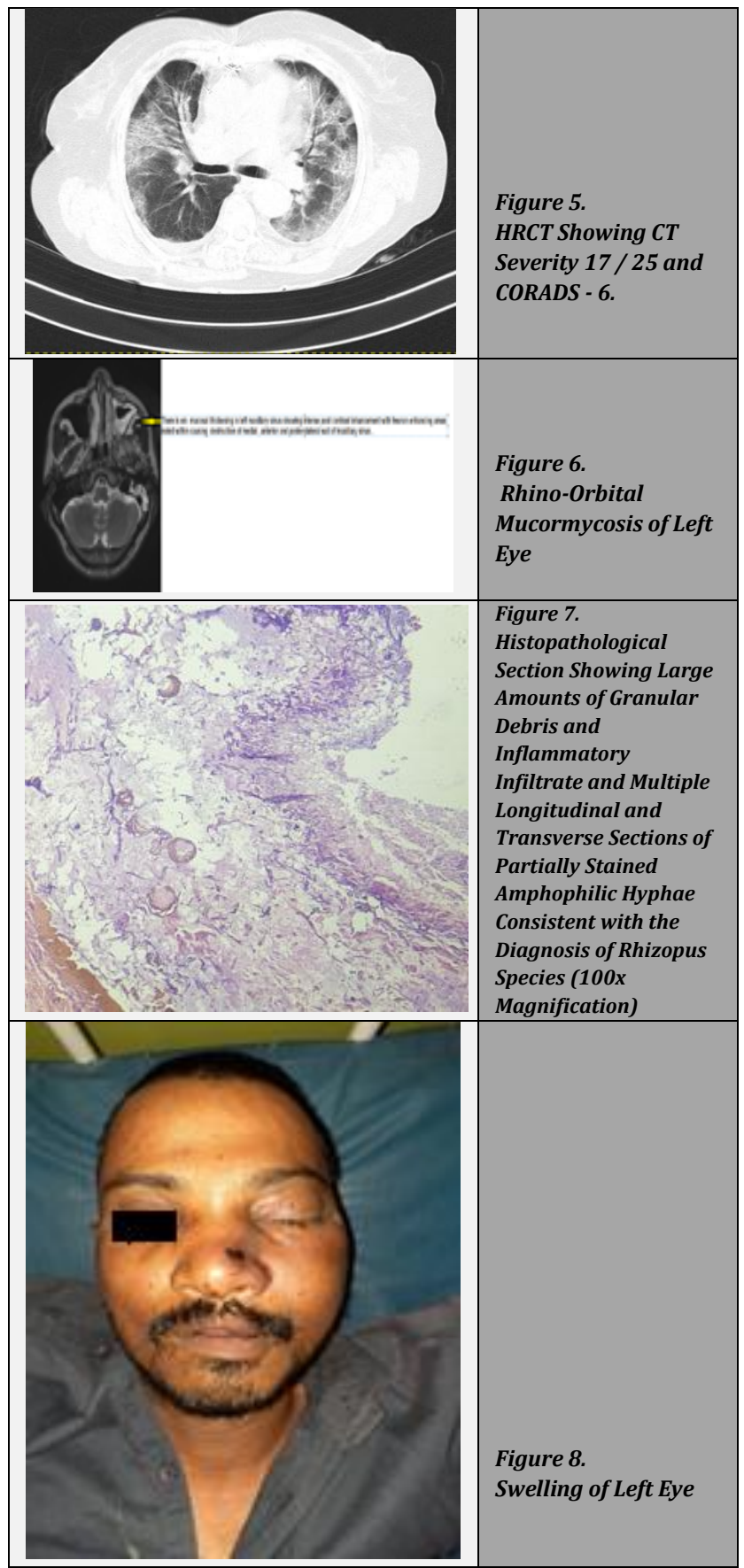


A 41 - year - old male presented with the chief complaints of breathlessness with dry cough along with swelling of the left eyelid which was profound.

The patient had no history of diabetes mellitus, hypertension or any other prior medical illness. On general examination, pulse was 74 beats per minute, regular rhythm, blood pressure was 140 / $88 \mathrm{~mm}$ of $\mathrm{Hg}$ in right arm supine position and Spo 2 was $89 \%$ on room air.

Local examination revealed extensive swelling over the left eyelid because of which the patient was unable to open the eye. Systemic examination revealed bilateral bronchial breath sound in the inframammary and mammary region, the rest of the systemic examination was unremarkable. HRCT Chest was done which was suggestive of bilateral ground-glass opacity with CT severity score of $17 / 25$ and CORAD 6 . Nasopharyngeal swab was sent for testing of COVID-19 which came positive by the RT PCR method.

The patient was started on remdesivir, oxygen support and other supportive measures. Laboratory investigations revealed a normal HbA1c and the patient was negative for HIV, Hepatitis B and Hepatitis C.

MRI paranasal sinus with orbit was done which was suggestive of rhino orbital mucormycosis. Debridement was done and a swab was sent for culture which showed growth of rhizopus species. The patient was started on liposomal amphotericin B. During the hospital stay, the patient improved clinically and was discharged on oral antifungals after 29 days of admission.

\section{Case 3}

A 68 - year - old male presented with a chief complaint of breathlessness, fever, swelling of the left eye along with blackish discolouration. There was a loss of vision in the left eye. The patient had a history of diabetes mellitus for 5 years and was on regular medication for the same with oral hypoglycaemics. There was no history of hypertension or tuberculosis in the past.

On general examination, pulse was 88 beats per minute, regular, blood pressure was 130 / $80 \mathrm{~mm}$ hg and Sp02 was 90 percent on room air. On local examination, there was profound swelling of the left eye along with blackish discolouration. Systemic examination revealed bilateral bronchial breath sounds present in the infra mammary region and the rest of the systemic examination was unremarkable. The patient was admitted and a nasopharyngeal swab was sent for RT PCR to test positive for COVID 19.

HRCT thorax was done and the patient had a score of $11 /$ 25 and Corads - 6. MRI paranasal sinus and orbit was done which showed features of rhino orbital mucormycosis. Debridement was done and the swab was sent for culture which showed growth of rhizopus species.

The patient was started on liposomal amphotericin B, remdesivir, oxygen support and other supportive measures. The patient improved clinically and was discharged after 40 days of admission on oral antifungal drugs.

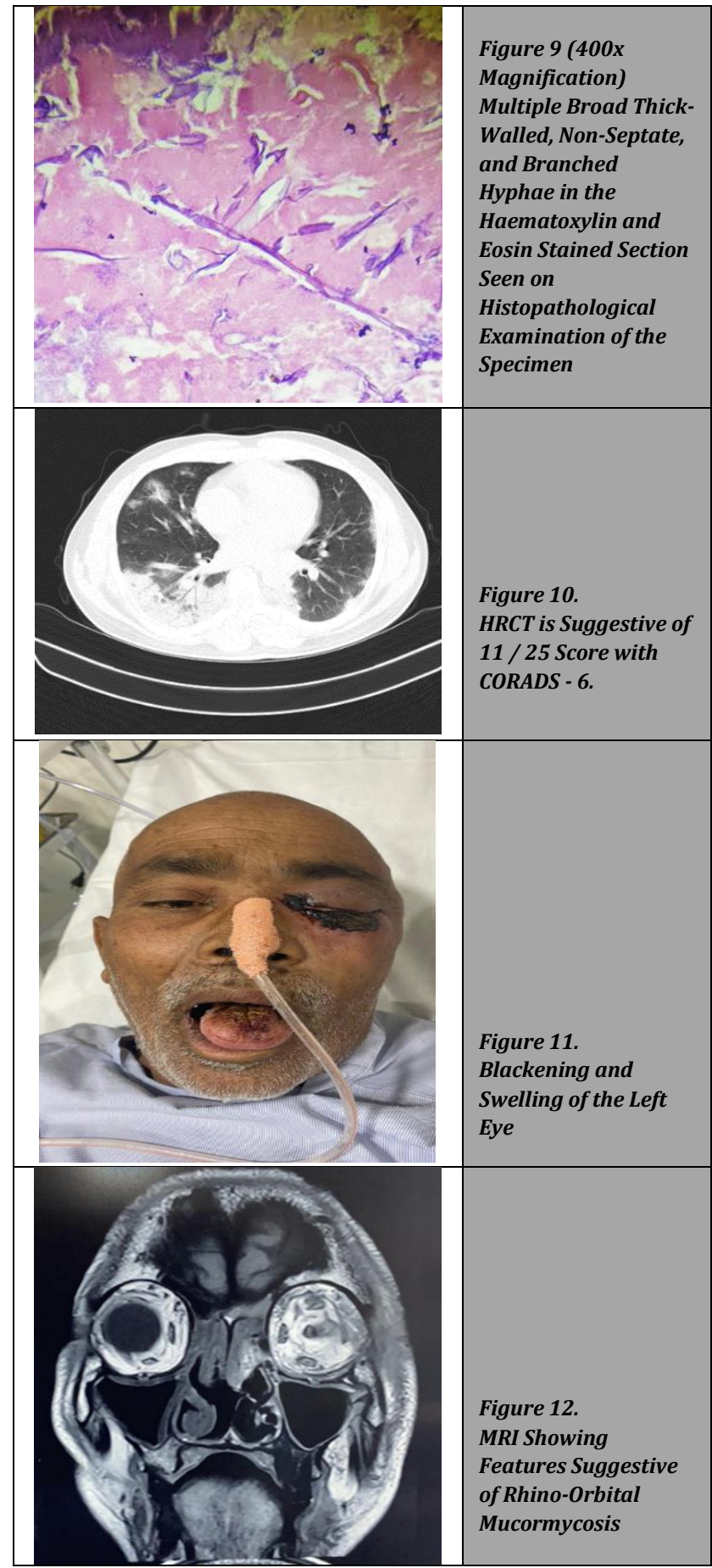

Case 4

A 56 - year - old male presented with the chief complaint of white coloured patch over his tongue and oral mucosa for 5 days. There was no history of diabetes mellitus, hypertension, tuberculosis or bronchial asthma in the past. On general examination, patient's pulse was 92 beats per minute, regular, blood pressure was 128 / $76 \mathrm{~mm}$ of $\mathrm{Hg}$ in the right arm supine position. On local examination, there was a white-coloured patch present over the tongue and oral mucosa suggestive of oral candidiasis. 
A swab was sent for culture from the lesion which showed the growth of candida species. The patient's nasopharyngeal swab was sent for RT PCR for COVID-19 which turned out to be positive. The patient was started on remdesivir, antifungal and other supportive measures. The patient's condition improved clinically and was discharged after 10 days of hospital stay.

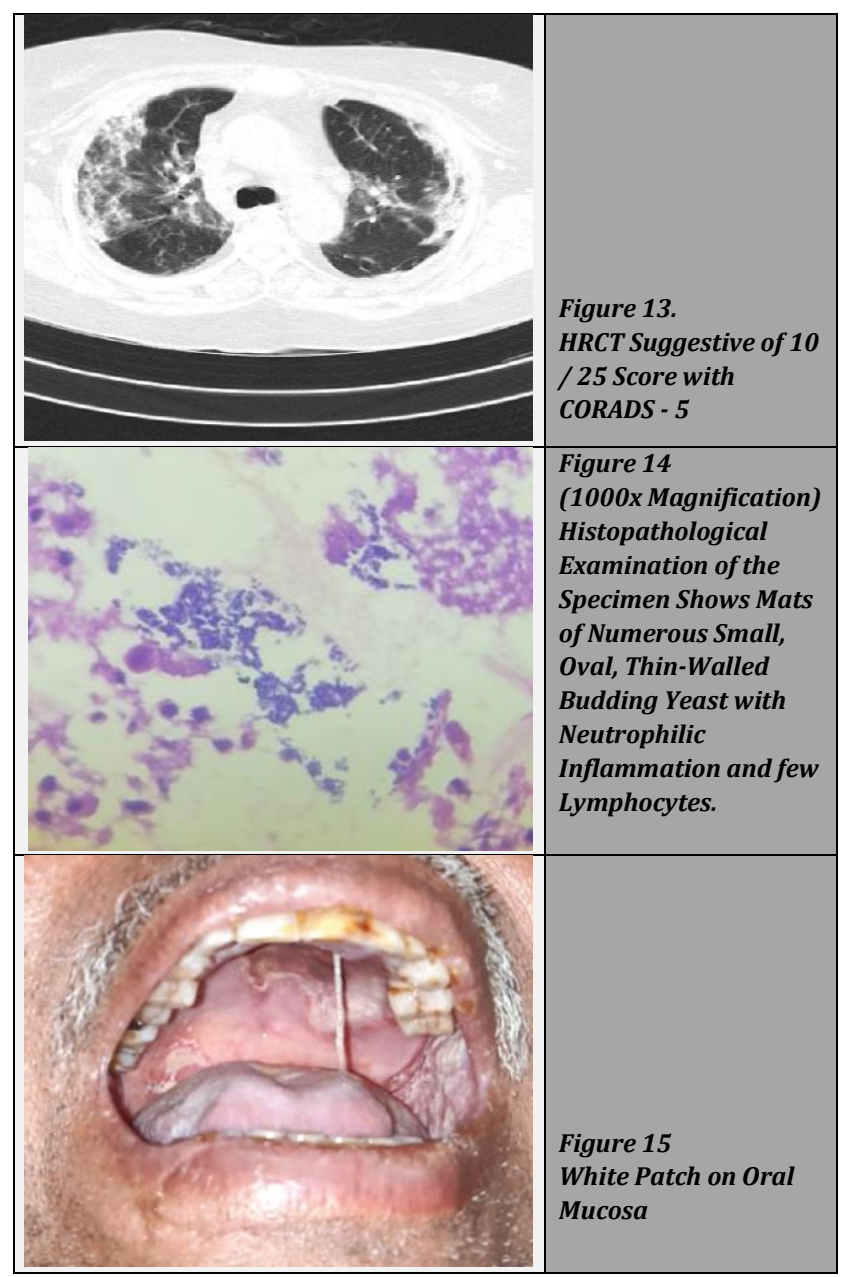

Case 5

A 39 - year - old male presented with fever, cough, and breathlessness with a whitish patch over the tongue along with oral mucosa for seven days. The patient was a known case of insulin dependent diabetes and was on rapid acting insulin for the same. There was no history of hypertension, bronchial asthma or any other chronic medical illness.

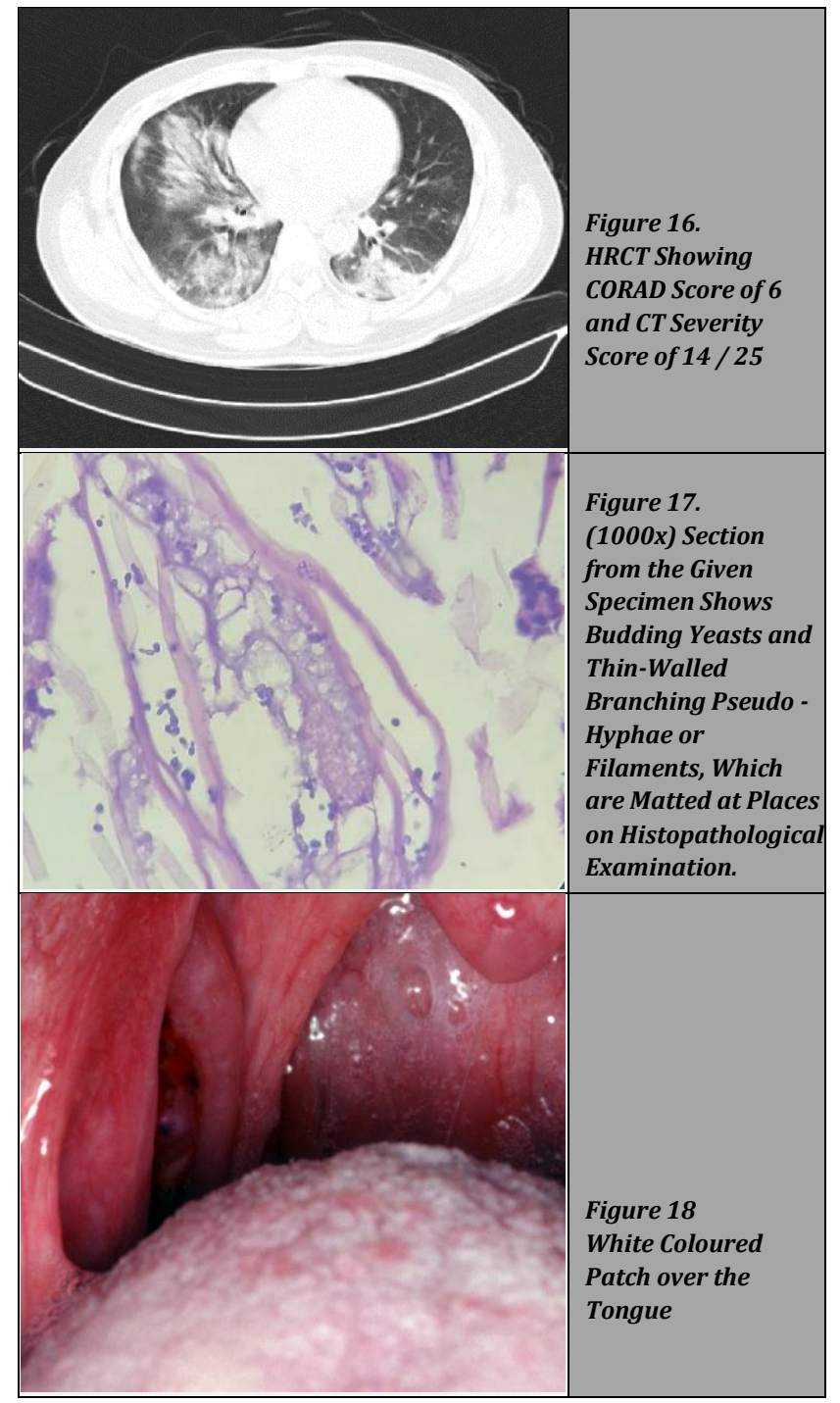

On general physical examination, pulse was 78 beats per minute, regular, blood pressure was 130 / 78 mm Hg in right arm supine position and SpO2 was $89 \%$ on room air. Local examination showed a white coloured patch over the tongue and oral mucosa. On systemic examination, chest was bilaterally clear, heart sounds were normal. The patient was conscious and oriented and the abdomen was soft and nontender.

\begin{tabular}{|c|c|c|c|c|c|}
\hline Lab Parameters & Case 1 & Case 2 & Case 3 & Case 4 & Case 5 \\
\hline $\mathrm{CBC}-\mathrm{Hb}$ & $10.2 \mathrm{gm} / \mathrm{dl}$ & $9.6 \mathrm{gm} / \mathrm{dl}$ & $11.8 \mathrm{gm} / \mathrm{dl}$ & $10.7 \mathrm{gm} / \mathrm{dl}$ & $13.4 \mathrm{gm} / \mathrm{dl}$ \\
\hline TLC & $10,400 / \mathrm{dl}$ & $12,600 / \mathrm{dl}$ & $14,000 / \mathrm{dl}$ & $9,900 / \mathrm{dl}$ & $16,300 / \mathrm{dl}$ \\
\hline Platelet count & $2.4 / \mathrm{dl}$ & $3.2 / \mathrm{dl}$ & $1.8 / \mathrm{dl}$ & $2.6 / \mathrm{dl}$ & $1.9 / \mathrm{dl}$ \\
\hline KFT - Creatinine & $0.9 \mathrm{mg} / \mathrm{dl}$ & $0.7 \mathrm{mg} / \mathrm{dl}$ & $1.20 \mathrm{mg} / \mathrm{dl}$ & $0.5 \mathrm{mg} / \mathrm{dl}$ & $\mathrm{mg} / \mathrm{dl}$ \\
\hline Urea & $36 \mathrm{mg} / \mathrm{dl}$ & $52 \mathrm{mg} / \mathrm{dl}$ & $44 \mathrm{mg} / \mathrm{dl}$ & $47 \mathrm{mg} / \mathrm{dl}$ & $39 \mathrm{mg} / \mathrm{dl}$ \\
\hline Sodium & $132 \mathrm{mmol} / \mathrm{l}$ & $142 \mathrm{mmol} / \mathrm{l}$ & $140 \mathrm{mmol} / \mathrm{l}$ & $139 \mathrm{mmol} / \mathrm{l}$ & $137 \mathrm{mmol} / \mathrm{l}$ \\
\hline Potassium & $4.2 \mathrm{mmol} / \mathrm{l}$ & $4.5 \mathrm{mmol} / \mathrm{l}$ & $3.9 \mathrm{mmol} / \mathrm{l}$ & $3.6 \mathrm{mmol} / \mathrm{l}$ & $4.1 \mathrm{mmol} / \mathrm{l}$ \\
\hline Inflammatory markers - CRP & $12.0 \mathrm{mg} / \mathrm{dl}$ & $26.0 \mathrm{mg} / \mathrm{l}$ & $44.0 \mathrm{mg} / \mathrm{dl}$ & $30.20 \mathrm{mg} / \mathrm{dl}$ & $25.8 \mathrm{mg} / \mathrm{dl}$ \\
\hline D - Dimer & 1.16 & 1.30 & 1.08 & 2.01 & 1.24 \\
\hline Serum & $1240 \mathrm{ng} / \mathrm{ml}$ & $1427 \mathrm{ng} / \mathrm{ml}$ & $1180 \mathrm{ng} / \mathrm{ml}$ & $892 \mathrm{ng} / \mathrm{ml}$ & $965 \mathrm{ng} / \mathrm{ml}$ \\
\hline \multicolumn{6}{|l|}{ Ferritin } \\
\hline IL - 6 & 21.0 & 16.0 & 13.0 & 41.0 & 18.0 \\
\hline HRCT score & $16 / 25$ & $17 / 25$ & $11 / 25$ & $10 / 25$ & $14 / 25$ \\
\hline CORADS & 6 & 6 & 6 & 6 & 6 \\
\hline HIV & Negative & Negative & Negative & Negative & Negative \\
\hline HBsAg & Negative & Negative & Negative & Negative & Negative \\
\hline HCV & Negative & Negative & Negative & Negative & Negative \\
\hline HbA1c & 7.6 & 5.9 & 9.1 & 6.2 & 8.4 \\
\hline
\end{tabular}


A nasopharyngeal swab was sent for RT PCR for COVID-19 which turned out to be positive. HRCT chest was done which showed bilateral ground-glass opacities with CORAD score of 6 and CT Severity Score of 14 / 25. A swab from the oral lesion was sent for culture which showed growth of candida. The patient was started on supplemental oxygen by face mask, remdesivir, oral fluconazole and other supportive measures. After 12 days of admission, the patient improved clinically and was discharged in stable condition. He is doing well on follow up.

\section{DISCUSSION}

With the steep increase in the global death burden of more than 3 million deaths caused by the lethal COVID-19 virus, a definite regimen for the management of COVID-19 has become a compelling necessity. Currently, with the available treatment protocols, antivirals are the foremost drugs given along with the glucocorticoids to control the ensuing inflammation caused by the cytokine storm produced by COVID-19 virus. Glucocorticoids is pivotal in preventing the development of hypoxia, the most dreaded complication of the virus and hence reducing the development of COVID associated viral pneumonia. Various immunosuppressive drugs such as tocilizumab has been reported to be promising in the management of the cytokine storm induced in COVID-19. These immunosuppressants and glucocorticoids together lead to altered immunity and predispose the patients to secondary opportunistic infections. Along with the various antivirals and immunomodulators, antibiotics have also been used in the management of COVID-19. This over judicious use of antibiotics results in an imbalance between the bacterial and fungal commensals leading to overgrowth of the fungal inhabitants. Diabetes mellitus in itself is a predisposing factor for fungal infections. ${ }^{3}$ Hyperglycation of iron - sequestering proteins disrupting the normal iron sequestration has been the likely mechanism to explain the development of mucormycosis infection in immunocompetent diabetic patients in COVID-19.4 Mucormycosis is a grave infection, however with early diagnosis and prompt action is greatly manageable but because of its aggressively spreading property carries a high risk of morbidity and mortality. ${ }^{5}$ Candidiasis is generally a common commensal of the human oral cavity but due to the altered immune status by the use of immunosuppressants and glucocorticoids and indiscriminate usage of antibiotics, it results in candidiasis infection. Thus, it is very essential to make prudent use of the available treatment modalities such as irge of the pandemic, its association with the opportunistic fungal infection documented currently could be just the tip of the iceberg. And hence judicious use of immunomodulators and glucocorticoids should be made. Thus, immunomodulators and glucocorticoids should be judiciously used for the management of patients.

\section{CONCLUSIONS}

Thus, as we struggle to fight a difficult battle with COVID-19 there is an emergence of newly reported opportunistic fungal infections with deadly complications further complicating this war with COVID-19. These fungal infections are turning out to be a "pandemic" hidden within the real pandemic of COVID-19 which is being neglected and needs more attention from the treating physicians in the form of intelligent use of corticosteroids and immunomodulator therapy.

Financial or other competing interests: None.

Disclosure forms provided by the authors are available with the full text of this article at jemds.com.

\section{REFERENCES}

[1] Jain A, Talwar D, Kumar S. Spectrum of respiratory involvement in COVID 19 era; an overview. Indian Journal of Forensic Medicine \& Toxicology 2020;14(4):6593-9.

[2] Jain S, Kumar S, Kaushal A. Rhinocerebral mucormycosis with isolated sixth nerve palsy in an immunocompetent patient. Med J Malaysia 2011;66(4):376-8.

[3] Rajeshwari A, Gangadhara Somayaji KS. Rhinocerebral Mucormycosis: an unusual presentation. Am J Med Sci 2012;2(1):16-9.

[4] Mehta S, Pandey A. Rhino-orbital mucormycosis associated with COVID - 19. Cureus 2020;12(9):e10726

[5] Revannavar SM, Supriya PS, Samaga L. COVID-19 triggering mucormycosis in a susceptible patient: a new phenomenon in the developing world? BMJ Case Rep 2021;14(4):e241663. 